\title{
Perceived area and the luminosity threshold
}

\author{
FREDERICK BONATO \\ Saint Peter's College, Jersey City, New Jersey \\ and \\ ALAN L. GILCHRIST \\ Rutgers University, Newark, New Jersey
}

\begin{abstract}
Observers made forced-choice opaque/luminous responses to targets of varying luminance and varying size presented (1) on the wall of a laboratory, (2) as a disk within an annulus, and (3) embedded within a Mondrian array presented within a vision tunnel. Lightness matches were also made for nearby opaque surfaces. The results show that the threshold luminance value at which a target begins to appear self-luminous increases with its size, defined as perceived size, not retinal size. More generally, the larger the target, the more an increase in its luminance induces grayness/blackness into the surround and the less it induces luminosity into the target, and vice versa. Corresponding to this luminosity/grayness tradeoff, there appears to be an invariant: Across a wide variety of conditions, a target begins to appear luminous when its luminance is about 1.7 times that of a surface that would appear white in the same illumination. These results show that the luminosity threshold behaves like a surface lightness valuethe maximum lightness value, in fact-and is subject to the same laws of anchoring (such as the area rule proposed by Li \& Gilchrist, 1999) as surface lightness.
\end{abstract}

Most surfaces in the visual world appear opaque. There are also surfaces that appear transparent and some that appear self-luminous. But, although self-luminous surfaces seem to attract our attention almost irresistably, the limited literature on this topic contains little insight into how we visually distinguish luminous surfaces from opaque.

Wallach (1948), on the basis of his classic work with disk/annulus displays, reported that a disk begins to appear luminous when it has a luminance value 2 to 3 times higher than that of a surrounding annulus. Lie (1977), working with essentially the same stimulus display, asked observers to increase the luminance of the center region until they felt confident that it could not be seen merely as a lighter shade of gray under the same illumination level as the annulus. To reach this value, which is closely related to what we have called the luminosity threshold, ${ }^{1}$ observers set the luminance of the target 4.1 times higher than that of the surround.

Ullman (1976) considered and rejected, largely on logical grounds, six candidate rules for identifying a luminous surface. According to Ullman, luminosity does not appear to be uniquely associated with any of these: (1) highest intensity in the field, (2) high absolute intensity, (3) local contrast, (4) global contrast, (5) intensity

The authors wish to acknowledge the support of the National Science Foundation (Grants DBS-9222104 and SBR 95-14679) and to thank Hal Sedgwick, Sten Sture Bergström, and an anonymous reviewer for their constructive comments. Correspondence should be addressed to F. Bonato, Department of Psychology, Saint Peter's College, 2641 Kennedy Blvd., Jersey City, NJ 07306 (e-mail: bonato_f@spcvxa.spc.edu).

-Accepted by previous editor, Myron L. Braunstein relative to average scene intensity, or (6) lightness computation. He proposed a new rule, according to which a surface is luminous if the luminance gradient across it is inconsistent with the gradient in the surrounding region.

In a previous paper (Bonato \& Gilchrist, 1994), we reported perhaps the most extensive experiments on luminosity perception. Our results support Ullman's rejection of the six rules. But, although we did not test Ullman's proposed rule directly, we obtained luminosity perception in the absence of Ullman's gradient consistency factor. Testing the luminosity threshold for targets standing in different levels of illumination, we found, not surprisingly, that there is no absolute luminance level associated with the luminosity threshold. Rather we found the luminosity threshold to vary with the illumination level. Speigle and Brainard (1996) found analogous results in the chromatic domain: The luminosity threshold for a target of a given color varies, in an ecologically valid way, with the color of the illumination.

Testing the luminosity threshold for a target with backgrounds of different reflectance, we found no consistent relationship between the local target:background luminance ratio and the luminosity threshold. On a white background, the target reached the luminosity threshold at a target:background ratio of $2.2: 1$, whereas the threshold occurred at a ratio of $9.5: 1$ for a middle gray background and a ratio of $63: 1$ for a black background.

In all of these conditions, we found one consistent fact: We always obtained a luminosity threshold at a luminance value 1.7 times higher than the luminance of a surface that would appear white in the immediate context. Moreover, our value of 1.7 is actually consistent with the 4.1 value obtained by Lie (1977). 
The solution to this paradox lies in an observation that had not been previously recognized. Increasing the luminance of a target can, in principle, have two opposite effects: either (1) the target can continue to appear white while the surrounding surfaces appear to become darker shades of gray or (2) the surrounding surfaces can appear to remain the same while the target (test field) gradually comes to appear luminous. We call these grayness induction (or downward induction) and luminosity induction (or upward induction), respectively, and both have been obtained in empirical work. Grayness induction is obtained in standard "brightness induction" experiments (Diamond, 1953; Fry \& Alpern, 1953; Heinemann, 1955; Horeman, 1965; Leibowitz, Mote, \& Thurlow, 1953; Torii \& Uemura, 1965). Increasing the luminance difference between a target (usually called the test field) and a neighboring region (called the inducing field) produces a perceived darkening of the darker region, but not a brightening of the brighter region. Freeman (1967, p. 173) notes that "inducing-field luminances less than test-field luminances have little effect on brightness judgments" of the test field. Although this darkening effect has been called brightness induction, we will call it grayness induction to distinguish it from the alternative of luminosity induction. We obtained luminosity induction (with no grayness induction) both when we tested the luminosity threshold in a complex laboratory scene (Bonato \& Gilchrist, 1994) and when we tested it in a disk/ Ganzfeld display (Gilchrist \& Bonato, 1995). In both cases, we found that increasing the luminance of the target (or decreasing the surround luminance) has virtually no darkening effect on the appearance of the surrounding laboratory or the surrounding Ganzfeld. The entire effect is absorbed by the target.

In disk/annulus displays, when the disk is an increment, one obtains both luminosity induction in the disk and grayness induction in the annulus at the same time. Consequently, in the Wallach (1948) and Lie (1977) studies, the annulus would have appeared gray, not white, when the disk reached the luminosity threshold.

We were able to determine that shade of gray because, in our disk/annulus experiments, we had obtained lightness matches for the annulus. When the Wallach and Lie ratios are corrected by taking into account this darkening of the annulus (plus Lie's $75 \%$ definition of threshold vs. our $50 \%$ definition), it turns out that their luminosity thresholds also occurred when the target was about 1.7 times higher than an annulus perceived as white.

The finding of a constant relationship between perceived white and the luminosity threshold implies that the luminosity threshold should be treated as a part of the lightness scale, in the same sense that one end of a ruler is part of the ruler.

In this paper, we extend our studies of luminosity, addressing several questions revealed by our previous work. The main question concerns the effect of the area of a target on its luminosity threshold. Recent work by $\mathrm{Li}$ and Gilchrist (1999) has shown strong effects of area on per- ceived lightness. If the luminosity threshold is part of the surface lightness dimension, then it should also show area effects. This implies that whether grayness induction, luminosity induction, or some combination is produced in a given display depends, rather simply, on the relative area of the lighter and darker parts of the display.

We have also investigated whether area effects depend on retinal area or perceived area, whether the luminosity threshold depends on the number of targets, and whether it depends on figure/ground properties.

\section{EXPERIMENT 1}

\section{Method}

Laboratory arrangements. The observers viewed one end of a laboratory room monocularly by looking through a pinhole in the center of a large, rigid partition. They saw either a square target surface $\left(2.0^{\circ}\right.$ square $)$, or a larger rectangular target surface $\left(7.2^{\circ} \times\right.$ $\left.9.5^{\circ}\right)$. The target appeared to be mounted in the center of a large $\left(56 \mathrm{~cm}\right.$ high $\times 64 \mathrm{~cm}$ wide, $\left.10.3^{\circ} \times 11.8^{\circ}\right)$ sheet of Color-Aid matte white (reflectance $=90 \%$ ) paper that was attached to the rear wall of the laboratory $3.1 \mathrm{~m}$ from the observer's eye. The observer's view is shown in Figure 1.

Although the target appeared to be coplanar with the large background, it was in fact only $71 \mathrm{~cm}$ from the observer's eye, mounted in the center of a large $(66 \mathrm{~cm}$ high $\times 122 \mathrm{~cm}$ wide $)$ sheet of clear glass. Because of the pinhole, both the edges of the target and the edges of the background were in focus at the same time. The glass was fixed in a vertical position but was rotated (clockwise as seen from above) $7^{\circ}$ away from parallel to the partition. This modest slant served to throw the reflection of the light source off to the right of the glass and also caused the right-hand edge of the target to touch the glass, preventing the back of the target from reflecting in the glass. Figure 2 shows the laboratory arrangement.

Each small square target was constructed of a $2.5-\mathrm{cm}$ piece of Color-Aid matte paper mounted on a thin wedge of aluminum. The aluminum wedge, which compensated for the slant of the glass, was mounted in the center of the glass sheet by means of an aluminum peg that protruded from the rear of the wedge and that fit snugly though a hole in the glass sheet. This peg allowed the experimenter to change targets quickly without touching the glass.

Each large rectangular target was a $9 \mathrm{~cm}$ high $\times 12 \mathrm{~cm}$ wide piece of Color-Aid matte paper mounted onto an identically sized piece of rigid cardboard. It was attached to the glass by means of a magnet and two aligning pins and, like the small target, was mounted in the observer's frontal plane using a wedge arrangement that compensated for the slant of the glass.

The only illumination was provided by a $250-\mathrm{W}$ quartz halogen bulb mounted immediately behind and $25 \mathrm{~cm}$ to the right of the viewing pinhole. Visibility of the light source was prevented by a metal baffle located between the pinhole and the bulb. In accordance with the inverse square law of illumination, this arrangement caused the targets on the glass to receive about 18 times more illumination than the white paper attached to the rear wall of the lab. More specifically, this arrangement caused a nearly black target (reflectance $=5 \%$ ) on the glass to have the same luminance as the white background attached to the rear wall of the lab. Because this physically black target also appeared white, this arrangement is an example of the famous lightness illusion first described by Gelb (1929). The target could be made to appear luminous simply by replacing it with one of a series of six targets representing increasing levels of reflectance.

The observer made lightness matches to the target's background by turning $90^{\circ}$ away from the viewing pinhole, to a grayscale of 16 Munsell chips mounted onto white cardboard, ranging from 


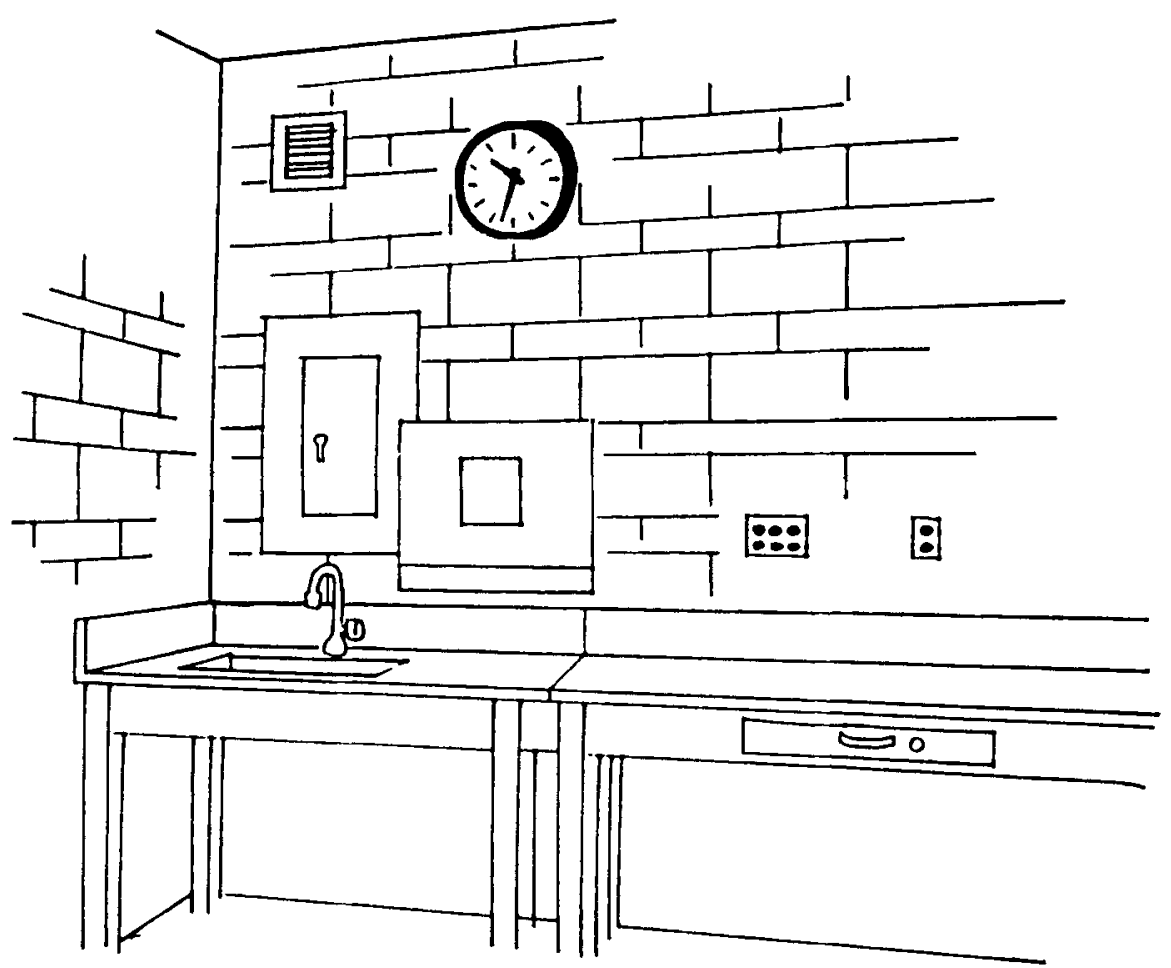

Figure 1. The visual field as seen by observers in Experiment 1.

black (reflectance $=3 \%$ ) to white (reflectance $=90 \%$ ), located to the left side of the observer and approximately $60 \mathrm{~cm}$ from the observer's eyes. The illumination on the chart was provided by a $15-$ W fluorescent bulb that produced a luminance value of $539 \mathrm{~cd} / \mathrm{m}^{2}$ for the white chip (Munsell 9.5, reflectance $=90 \%$ ).

Design. One group of 14 observers judged the small square targets, and a separate group of 14 judged the larger rectangular tar- gets. The reflectance values of the six targets were the same for both groups, $8.5,15,23,30,35$, and 50 , with corresponding luminance values of $25,45,69,89,106$, and $151 \mathrm{~cd} / \mathrm{m}^{2}$. The white background had a reflectance of $90 \%$, with a corresponding luminance value of $16 \mathrm{~cd} / \mathrm{m}^{2}$. Each observer served in six trials, one for each reflectance value, in random order. In addition, the observers matched the lightness of the background on each trial.

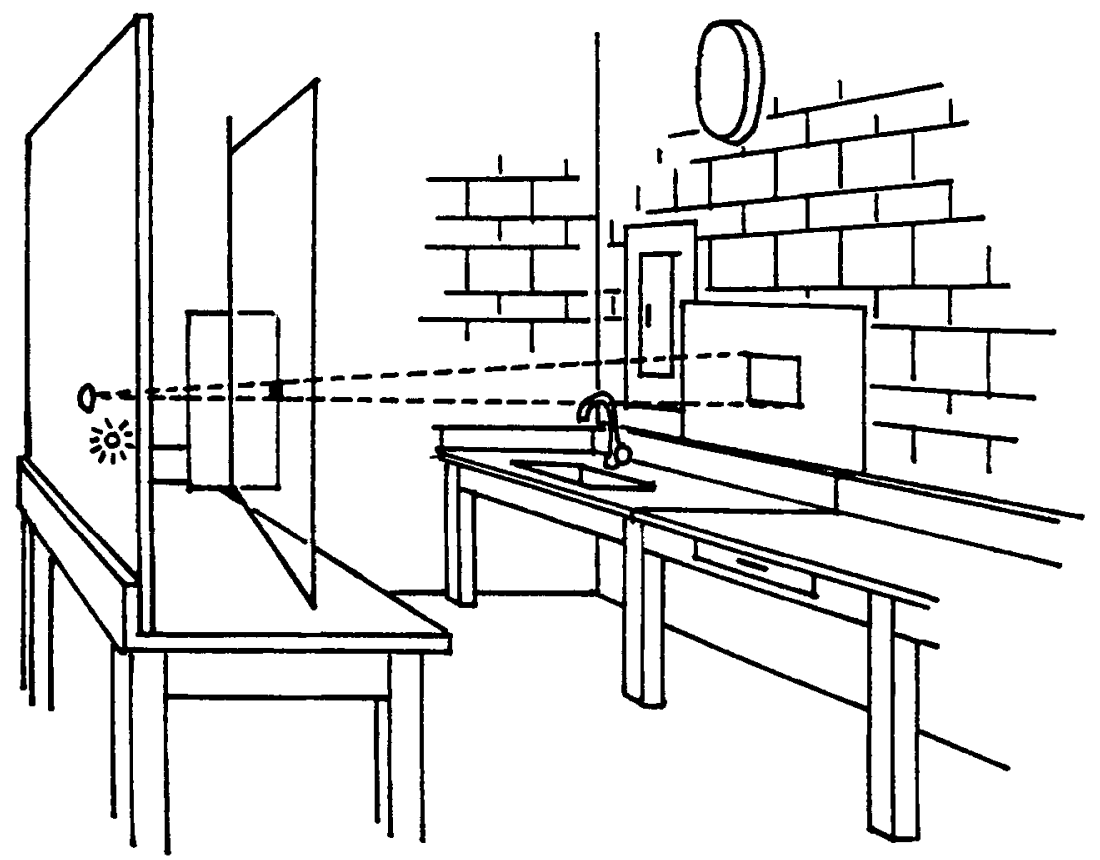

Figure 2. Laboratory arrangements for Experiment 1. 
Procedure. The observer was led into the laboratory and seated in front of the viewing pinhole. The experimenter then set the lighting conditions and read the following instructions:

In this experiment you will be asked to make judgments about whether or not a surface appears to be glowing - by glowing I mean that the surface appears too bright to simply be a piece of white paper. It might appear to be emitting light from within, like a light source, or it might appear to be a special beam of illumination. When looking through the pinhole in front of you, you will notice a large rectangular shaped area on the far wall underneath the clock. The target area to be judged will always be the square (or rectangular) patch that is embedded within this large rectangle. After each presentation of the target you will tell me if the target appears to be glowing or not glowing. Immediately after making this judgment I want you to imagine taking a piece of the target's background off the wall and placing it against the chart that is to your left. I then want you to tell me which chip the background paper would most closely blend in with. Do you have any questions?

If the observer had no questions the experimenter opened a shutter occluding the pinhole and :irected the observer to look through the pinhole, locate the target, make a forced-choice luminosity judgment by saying either "glowing" or "not glowing," and make a lightness match to the target's background. The experimenter then closed the shutter, recorded the observer's response, changed the target on the glass, and opened the shutter, thereby marking the beginning of the next trial. If an observer perceived no targets to be luminous, an additional target with a luminance value of $271 \mathrm{~cd} / \mathrm{m}^{2}$ was presented at the end of the experiment. The purpose of presenting this target was to determine whether or not the observer's failure to report luminosity was due to a misunderstanding of the instructions.

Observers. Twenty-eight naive undergraduates volunteered to serve as observers. The observers were students with a median age of 19 years, almost equally divided between male and female, with normal or corrected-to-normal vision. They volunteered to complete a class requirement and were naive as to the purpose of the experiment.

\section{Results and Discussion}

The percentage of luminosity reports as a function of the luminance of the target is shown in Figure 3. A comparison of the curves for small and large targets clearly suggests that as the area of a surface is increased, its luminosity threshold also increases.

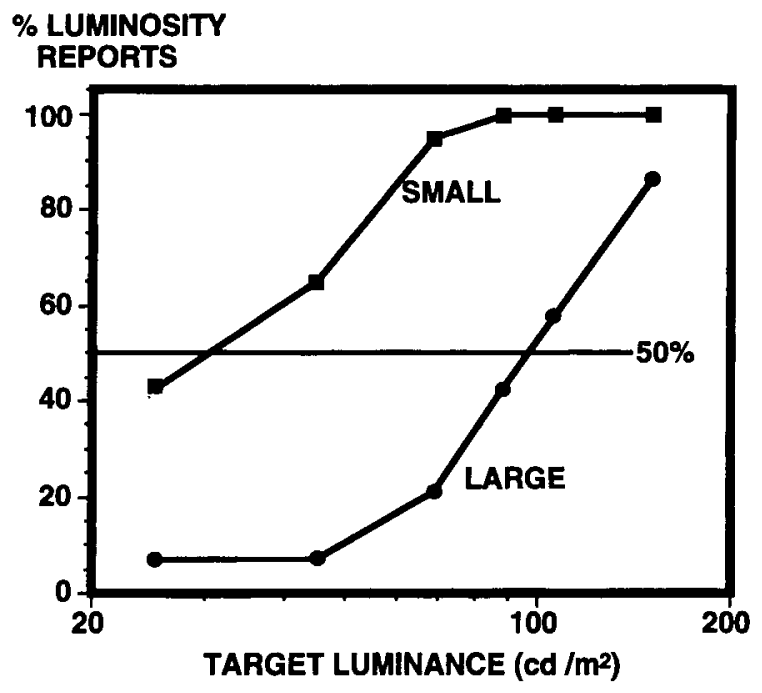

Figure 3. Percentage of luminosity reports obtained in Experiment 1 as a function of target luminance.
It might be noted that we did not take our threshold measures from this graph, however, but calculated luminosity thresholds in the following way. A threshold value was obtained for each observer by calculating the arithmetic mean of the following two values: (1) the highest luminance level at which the target region was seen as not luminous with no luminosity reports for any lower values, and (2) the lowest luminance level at which the target region was seen as self-luminous and was seen so consistently at higher luminance levels. The mean threshold for the large target was $101 \mathrm{~cd} / \mathrm{m}^{2}$, significantly higher $[t(26)=5.3, p<.001]$ than the threshold of $34.6 \mathrm{~cd} / \mathrm{m}^{2}$ obtained for the small target. In fact, 2 of the observers failed to perceive any of the large targets as self-luminous. ${ }^{2}$ To calculate a threshold for these observers, we used the arithmetic mean of $151 \mathrm{~cd} / \mathrm{m}^{2}$, which was the highest target luminance presented in the experiment, and the luminance of the next brightest target, $271 \mathrm{~cd} / \mathrm{m}^{2}$, had one been presented. If anything, this method tends to underestimate the threshold value.

The lightness judgments for the target's background are shown in Figure 4. As the luminance of the smaller target was increased, the mean Munsell match to the background remained relatively unchanged and appeared as a light gray (reflectance $=64 \%$ ). As the luminance of the large target was increased, the mean Munsell match to its background decreased significantly $[F(5,65)=38$, $p<.01]$, from a light gray (reflectance $=54 \%$ ) to about a middle gray (reflectance $=26 \%$ ). This suggests that, under these conditions, there is a systematic relationship among three variables: the area of the target, the target:background ratio required for luminosity, and the lightness of the background. ${ }^{3}$

We see a kind of invariance here. The ratio between the luminance value at the luminosity threshold and the luminance of a perceived white remains constant. If the luminosity threshold is measured in terms of the target:background luminance ratio, then we obtain one threshold ratio for the small target $(2.2: 1)$ and a very different threshold ratio for the large target (6.3:1).

But the background did not appear to be the same shade of gray at threshold in the two cases. Taking this fact into account, we can compute a ratio between target luminance at threshold and the luminance of a background that would be perceived as white. For example, at the luminosity threshold for the large target, the background is perceived as having a reflectance of $26 \%$. This value is 3.5 times darker than the reflectance of a perceived white (i.e., $90 \%$ ). If we divide our target:background threshold ratio of $6.3: 1$ by this value of 3.5 , we obtain a ratio of $1.8: 1$, which represents the luminance ratio between the threshold and perceived white. Applying the same transformation to the 2.2:1 threshold obtained with the small target, we obtain a ratio of $1.6: 1$, nearly the same.

These results are consistent with those we obtained in our previous experiment (Bonato \& Gilchrist, 1994) using the same apparatus as the one used in Experiment 1 with only slight modifications. In that experiment, the target's 


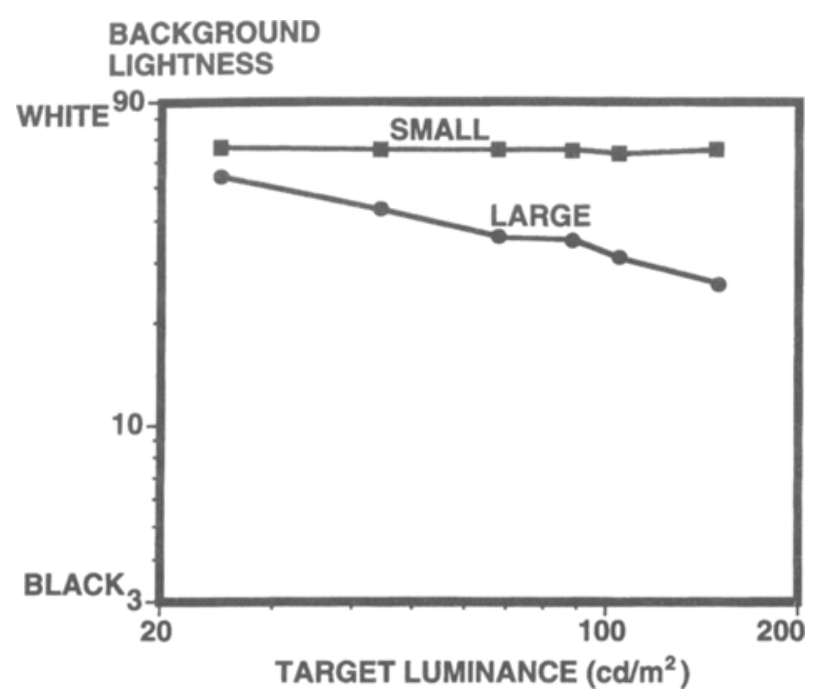

Figure 4. Lightness judgments for the background of the target in Experiment 1.

background was black (reflectance $=3 \%$ ), gray (reflectance $=19 \%$ ), or white (reflectance $=90 \%$ ). But, for all three backgrounds, when we calculated the luminance of a background that would have been perceived as white (using the correction described above), we found a luminosity threshold 1.6 times higher than that value.

These results appear to provide a basic answer to the direction-of-induction question: When does increasing the luminance difference between a target and its surround produce luminosity induction, and when does it produce grayness induction? For the small target, the increasing luminance difference is almost entirely attributed to the target, which quickly becomes luminous. But, for the large target, less of the increasing luminance difference is attributed to the target and more to a darkening of the background, requiring a higher luminance to reach threshold. All of this is consistent with the area rule proposed by $\mathrm{Li}$ and Gilchrist (1999).

It is possible that our results do not mean that large targets have a higher luminosity than small targets but rather that regions perceived as background have a higher threshold than regions perceived as figure. Several investigators (Goldhammer, 1934; Künnapas, 1957; Oyama, 1950) have shown that decreasing the size of a region causes it to appear more as figure and less as ground. Coren (1969) has claimed a surface perceived to be figure exhibits more contrast (relative to its surround) than when it is perceived to be ground. Thus, it may be that a smaller target, appearing more figure-like, appears brighter than a larger target of the same luminance, reaching the luminosity threshold sooner. This hypothesis was tested in Experiment 2.

\section{EXPERIMENT 2}

In Experiment 2, we placed a small square figure in the center of the large target used in Experiment 1 to see whether this would further raise the luminosity threshold by making the large target appear more ground-like.

\section{Method}

The method was identical to that used in Experiment 1 with the following exceptions: (1) The white Color-Aid paper that covered the plywood board was removed so that the target's immediate surround was bare wood. (2) Only large targets were presented. (3) In one condition a $2.5-\mathrm{cm}^{2}$ (square) decremental square of Color-Aid paper was mounted in the center of each target. In each case, this square was chosen so as to have a reflectance value approximately $50 \%$ lower than the target upon which it was mounted. (4) The instructions given to the observer were modified in order to make it clear that the surface to be judged for luminosity was the rectangular region that surrounded the embedded square, and not the embedded square itself. (5) The observers were not required to make Munsell matches to the target's background. (6) One group of $10 \mathrm{ob}-$ servers judged targets with embedded squares, and a separate group of 10 judged targets without embedded squares.

\section{Results and Discussion}

The percentage of luminosity reports plotted as a function of the luminance of the target is presented in Figure 5. Clearly, the target containing the figure did not show a higher threshold, as it would if regions that are perceived as ground are less likely to be perceived as luminous. Noguchi and Kozaki (1985) found an analogous result in the Gelb effect. If anything, the target containing the figure showed a lower threshold. Although the difference between the means of the individual thresholds did not reach significance $[t(18)=0.38]$, one can clearly see in Figure 5 that the target containing the figure produced a larger percentage of luminosity reports than did the homogeneous target, for all six target luminance values.

Whether this outcome is consistent with our results of Experiment 1 depends on how area is defined. Area can refer either to the area of the retinal image produced by a surface or to the perceived area of a surface (Rock \& Brosgole, 1964; Rock \& Ebenholtz, 1959). If retinal area

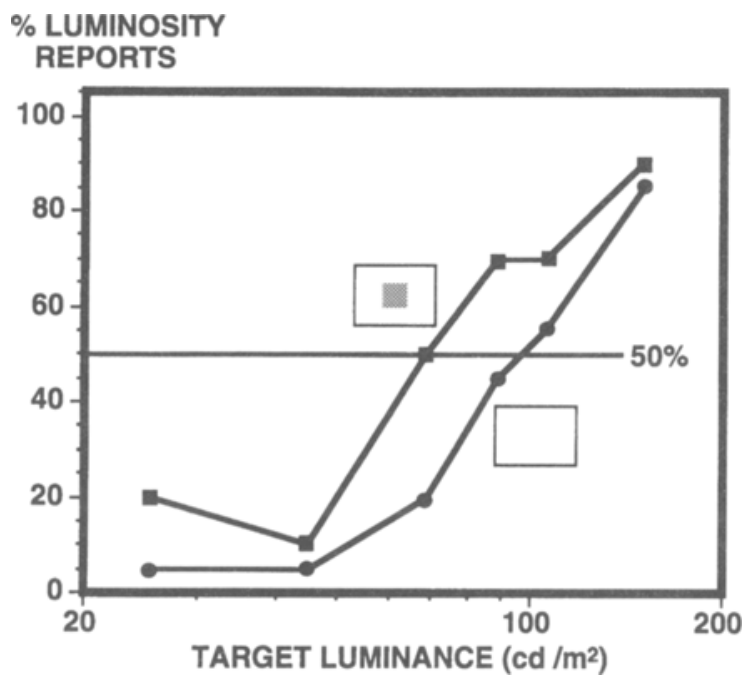

Figure 5. Percentage of luminosity reports obtained in Experiment 2 as a function of target luminance. 
is critical, then placing a figure on the target should lower its threshold because the figure obscures some of the retinal area of the target, and this would be consistent with the results of Experiment 1. On the other hand, if perceived area is critical, and if placing a figure on the target makes no change in its perceived area, one would expect the target with the figure to show the same threshold as the target without the figure. However, Kanizsa (1979) has shown that there is a perceptual shrinkage of the occluded (amodal) region of a surface, and Shimojo and Nakayama (1990) have shown analogous results using an apparent motion display. Thus, placing a figure on the target would reduce its perceived area some (but not as much as this would reduce its retinal area), producing a slightly lower threshold, according to the results of Experiment 1.

In Experiment 3, we tested whether the luminosity threshold depends on retinal area or perceived area.

\section{EXPERIMENT 3}

In Experiment 3, we presented a center/surround display in a dark environment, varying distance and overall display size in one condition, so as to change perceived area while keeping retinal area constant, and varying distance only in the other condition, so as to change retinal area while keeping perceived area constant.

\section{Method}

Laboratory arrangements. The laboratory arrangements are shown in Figure 6. The observer was presented with a center/surround display that consisted of a cardboard square, either $15 \mathrm{~cm}$ or $45 \mathrm{~cm}$ on a side, covered with Color-Aid matte gray paper (reflectance $=$ $19 \%$ ), centered within which was a small square aperture that served as the target. For the $15-\mathrm{cm}$ display, the target was $1.9 \mathrm{~cm}$ on a side; for the $45-\mathrm{cm}$ display, the target was $5.7 \mathrm{~cm}$ on a side. The background square was attached to the near end of a rectangular tunnel, $10 \mathrm{~cm}$ wide $\times 10 \mathrm{~cm}$ high $\times 61 \mathrm{~cm}$ long. The surface that appeared to fill the target aperture and that appeared coplanar with the background square was part of a gray square of Color-Aid matte paper (reflectance $=19 \%$ ) that was $6.4 \mathrm{~cm}$ across and was located inside the tunnel $13 \mathrm{~cm}$ behind the target aperture. It was supported by a rectangular sheet of transparent acrylic. Light was introduced into the tunnel from its far end by a Kodak Carousel slide projector. The interior of the tunnel was painted matte white for the purpose of light diffusion. The luminance of the target was controlled by sliding rectilinear cardboard panels containing holes of various diameter into a position $40 \mathrm{~cm}$ in front of the projector lens at the far end of the chamber. The background square was illuminated from the front by two additional Kodak Carousel projectors positioned $55^{\circ}$ from the surface normal, on opposing left and right sides, and each $2.5 \mathrm{~m}$ from the center of the display.

The observer stood in a viewing booth and looked binocularly through a horizontal slot at the display, which was located $0.47 \mathrm{~m}$, $1.4 \mathrm{~m}$, or $4.2 \mathrm{~m}$ beyond the slot. The $15-\mathrm{cm}$ display, when viewed from $1.4 \mathrm{~m}$, produced the same retinal image (target, $0.77^{\circ}$; background, $6.2^{\circ}$ ) as the $45-\mathrm{cm}$ display viewed from $4.2 \mathrm{~m}$. The $15-\mathrm{cm}$ display, when viewed from $0.47 \mathrm{~m}$, produced visual angles of $2.3^{\circ}$ for the target and $18^{\circ}$ for the background. The relative sizes of the

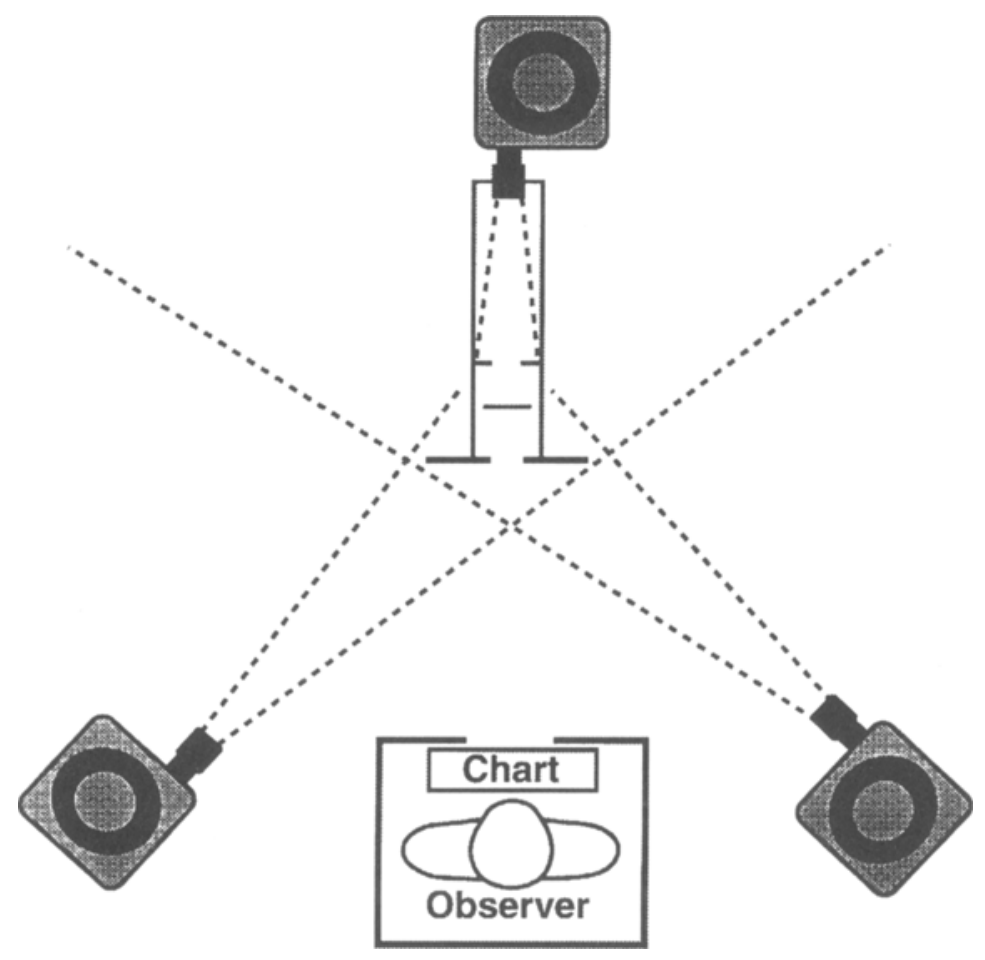

Figure 6. Laboratory arrangements for Experiment 3. 
target and the background were the same in all conditions. The same Munsell scale used in Experiment 1 (with the same level of illumination) was placed on a shelf just below the viewing slot, within the booth. We ensured that the observer's entire visual field, except for the display and the Munsell chart, was totally dark by using the viewing booth and by covering surfaces in the laboratory with either black paint or black cloth.

Design. In the standard condition, one group of 16 observers viewed the $15-\mathrm{cm}$ display at the $1.4-\mathrm{m}$ viewing distance. In the retinally larger condition, a second group of 15 observers viewed the same $15-\mathrm{cm}$ display at the $0.47-\mathrm{m}$ viewing distance. In the perceptually larger condition, a third group of 15 observers viewed the 45 $\mathrm{cm}$ display at the $4.2-\mathrm{m}$ viewing distance. For all three conditions, luminance values for the target were $21,37,62,103,192$, and $343 \mathrm{~cd} / \mathrm{m}^{2}$. The luminance of the background was always $21 \mathrm{~cd} / \mathrm{m}^{2}$. Each observer was presented with every target luminance value, once each, in random order.

Procedure. Each observer was led into the darkened laboratory, told to step into the viewing booth, and then read the following instructions:

In a moment I will ask you to look through the slot in front of you and you will see a small square region surrounded by a larger square. I want you to make a forced-choice judgment as to whether or not the small square is glowing. By glowing I mean that the square is too bright to simply be a piece of white paper. It might appear to be emitting light from within, like a light source. By glowing, I also mean that the small square may appear to be a special beam of illumination. I then want you to decide what color paper you think the larger square is made of. You will indicate your choice using the chart of color chips numbered from 2 to 9.5 which is on the shelf in front of you. In other words, I want you to imagine taking a piece of the larger square off the display and bringing it back here to the booth. If you placed the piece against the chart, which chip would it most closely blend in with? After deciding on your match, simply call out the number below the chip that you have decided on. After each trial there will be nothing visible through the slot. There will be six trials in all. Do you have any questions?

If the observer had no questions, the slot was opened by means of a shutter, marking the beginning of the first trial. The observer then made a forced-choice luminosity judgment for the target and a lightness match to the background. This was repeated for all target luminances. After all trials were completed, if the observer had seen no target as luminous, the experimenter increased the lumi-

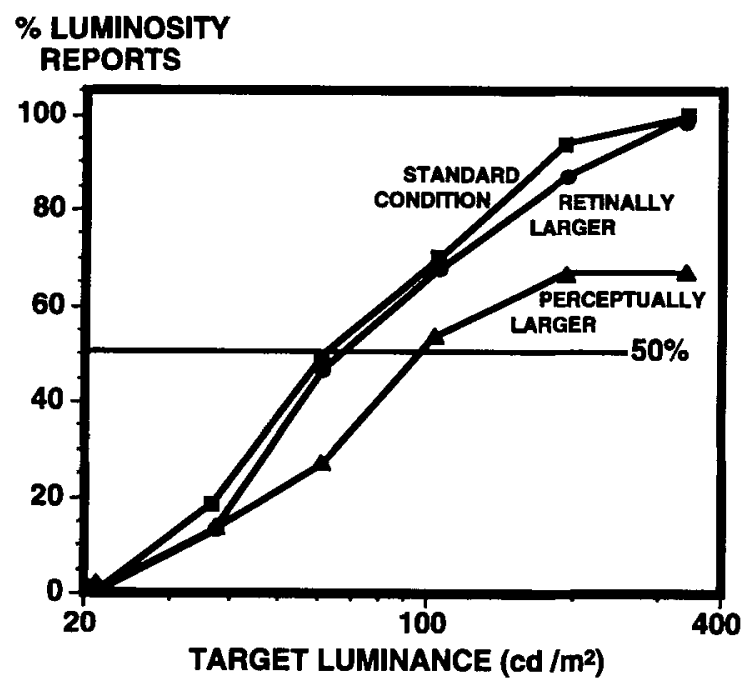

Figure 7. Percentage of luminosity reports obtained in Experiment 3 as a function of target luminance. nance of the target to $676 \mathrm{~cd} / \mathrm{m}^{2}$ and then asked the observer to make an additional forced-choice luminosity judgment. The observer was then shown a $0.9-\mathrm{m}$ ruler and asked to indicate on the ruler the apparent size of both the target and the background. The experimenter then interviewed the observer regarding his or her visual experience and revealed the set-up.

Observers. Forty-six naive undergraduates volunteered to serve as observers in order to fulfill a course requirement.

\section{Results and Discussion}

The percentage of luminosity reports plotted as a function of target luminance is shown in Figure 7. Four of the observers in the perceptually larger condition perceived no targets as luminous. ${ }^{4}$

Individual threshold values were obtained in the same manner as described for Experiment 1. An analysis of variance indicated that there was a significant difference among the mean individual thresholds $(F=3.47, p=$ .04). A Tukey's HSD post hoc test indicated that the mean luminosity threshold obtained for the perceptually larger condition was significantly higher than the mean threshold obtained for the standard condition, even though the retinal images of these two displays were identical. The target in the perceptually larger condition was estimated at $8.1 \mathrm{~cm}$, significantly larger $[F(2,42)=14.2, p<.001]$ than the estimate of $2.3 \mathrm{~cm}$ for the target in the standard condition.

The threshold obtained for the retinally larger condition was not significantly different from that obtained for the standard condition. The retinal images of these displays were different, but their apparent sizes were very much alike. The target in the retinally larger condition was estimated at $2.0 \mathrm{~cm}$, not significantly different from the estimate of $2.3 \mathrm{~cm}$ for the target in the standard condition.

Lightness judgments for the backgrounds are shown in Figure 8. There is very little difference between the three curves. Thus, these results do not show the close coupling between the luminosity threshold and background lightness that we found in our other experiments. If the luminosity threshold is always 1.7 times the luminance of a perceived white surface, then a larger target should not only push the threshold higher but also make the background appear darker. We do not have a clear explanation for this result.

\section{EXPERIMENT 4}

Experiment 1 showed that increasing the size of a single surface both increases its luminosity threshold and decreases the lightness of other surfaces present in the same illumination. Experiment 4 was designed to test whether or not the presentation of multiple targets, whose combined visual area was greater than that of a single target, would also yield the same pattern of results.

\section{Method}

Laboratory arrangements. The stimulus, which was viewed from a distance of $51 \mathrm{~cm}$, consisted of a pair of $10-\mathrm{cm}^{2}$ achromatic Mondrians $\left(18^{\circ} \times 12^{\circ}\right)$ joined to form a $90^{\circ}$ dihedral angle and sus- 


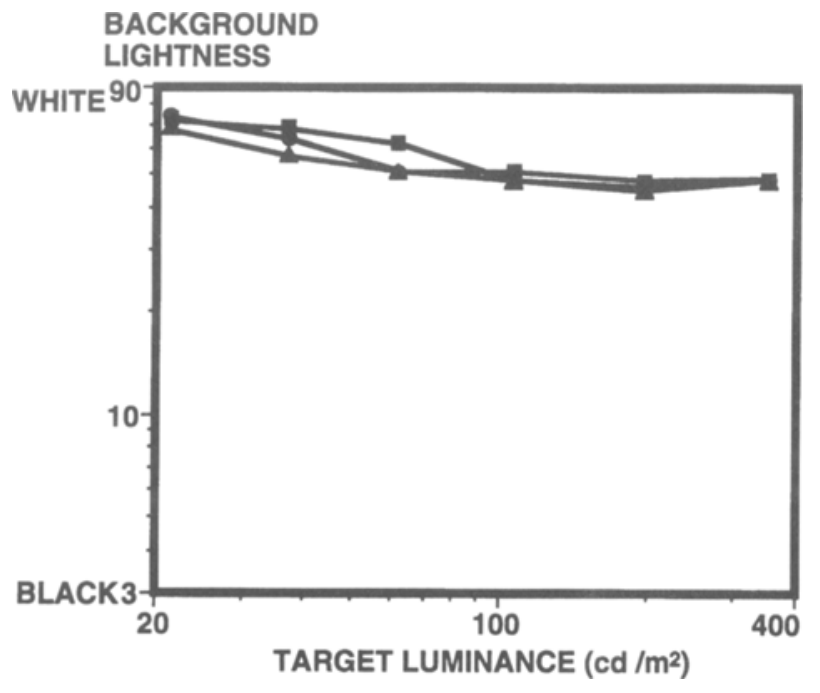

Figure 8. Lightness judgments for the background of the target in Experiment 3.

pended in the center of a vision tunnel ${ }^{5}$ by means of a horizontal rod. The rod, hidden from the observer by the Mondrians, was anchored to the center of a square translucent panel $66 \mathrm{~cm}$ behind the Mondrians that served as the back wall of the tunnel. Rear illumination of the panel was provided by a 150 -W floodlight bulb positioned $76 \mathrm{~cm}$ behind the panel; it produced a homogeneous background of $192 \mathrm{~cd} / \mathrm{m}^{2}$ for the display, with no shadow of its supporting rod.

The illumination for each Mondrian came through a window in the side wall of the tunnel from a $150-\mathrm{W}$ floodlight bulb controlled by a rheostat and located $48 \mathrm{~cm}$ from the Mondrian. The Mondrian on the right received 30 times more illumination than the Mondrian on the left.

Each Mondrian was composed of about 20 rectilinear pieces of achromatic matte Munsell paper randomly distributed. Six reflectances were used: $3 \%$ (black), $9 \%, 20 \%, 36 \%, 59 \%$, and $90 \%$ (white). Each of these reflectances appeared three or four times on each Mondrian. The four targets, although appearing to be coplanar with other areas on the left-hand Mondrian, were actually carefully cut apertures approximately $2.3^{\circ}$ square. The light that filled these apertures was reflected from a $7.5 \times 17.5 \mathrm{~cm}$ panel of Color-Aid matte paper mounted behind the aperture in a holder at a $45^{\circ}$ angle to the line of sight. This panel was illuminated by a $500-W$ incandescent bulb located just above a $5 \times 20 \mathrm{~cm}$ aperture cut out from the ceiling of the tunnel.

The observer sat in a viewing booth at the near end of the tunnel and viewed the display through a pair of $2.5-\mathrm{mm}$-diameter circular apertures, each of which was located in a $3.7-\mathrm{cm}$-diameter plastic hemisphere fashioned from a ping-pong ball half mounted in a sliding panel. This allowed the distance between the two apertures to be adjusted to match the interocular distance for each observer. Parallel sliding panels served as shutters. Figure 9 shows the display as seen by the observer. A line drawing of the display, with regions marked by numbers, was located on a slanted shelf just above the observer's lap. This drawing and a Munsell chart on the same shelf were both illuminated by a hidden 20 -W fluorescent bulb located $25 \mathrm{~cm}$ above the shelf. The chart consisted of sixteen $1.27 \times$ $2.54 \mathrm{~cm}$ chips that ranged from Munsell 2.0 (reflectance $=3 \%$ ) to 9.5 (reflectance $=90 \%$ ), all of which were mounted on a $10 \times$ $30 \mathrm{~cm}$ piece of white cardboard. The luminance of the white chip (reflectance $=90 \%$ ) was $117 \mathrm{~cd} / \mathrm{m}^{2}$.

Design. The six target panels had reflectance percentages of 17 , $29,41,53,70$, and 90 , with corresponding luminance values of 34 ,
$58,82,106,141$, and $182 \mathrm{~cd} / \mathrm{m}^{2}$. Each observer saw all six target values once in random order.

Procedure. The observer was led into the laboratory and seated in the viewing booth. The viewing holes were adjusted for the observer's interocular distance, the room lights were turned off, and the following instructions were read:

\begin{abstract}
In this experiment you will be asked to do two things. Your first task will be to make judgments about whether or not a surface appears to be glowing. By glowing I mean that the surface appears too bright to be a piece of white paper; for example, it may appear to be emitting light from within. If look down you will see a drawing of the display you are about to see through the eye holes in front of you. When I call out a number, I want you to locate that region on the drawing in front of you, locate the region on the display that corresponds to it, and tell me whether or not that region is glowing. You can check back to the drawing at any time should you feel the need to do so.

In addition to this task, on each trial I will ask you to make color matches to twelve other areas on the display. Again, I will call out a number, you will locate it on the drawing, locate the region on the display which corresponds to it, and then decide what color paper that region is made out of. Imagine taking that region off the display and placing it against the chart above your lap, which chip would the piece of paper most likely blend in with? Immediately after each trial there will be a brief rest period during which nothing will be visible through the eye holes. There will be six trials in all. Do you have any questions?
\end{abstract}

If there were no questions, the experimenter opened the shutters and began the first trial. The observer was asked to make a forcedchoice luminosity judgment for a randomly chosen aperture on the display and to make lightness judgments for 12 other surfaces on the display. Surfaces for which lightness judgments were taken were chosen randomly, and each reflectance value was probed twice on each trial. The experimenter then closed the shutters, changed the target panel, and reopened the shutters, marking the beginning of the next trial. After all six trials were completed, the observer was asked about his or her visual experiences of the stimuli in the experiment, and the set-up was then revealed.

Observers. Ten naive undergraduates volunteered to serve as observers in order to fulfill a course requirement.

\section{Results and Discussion}

We compared the luminosity threshold obtained for the four targets in this experiment with that obtained for a single target taken from an earlier experiment we con-

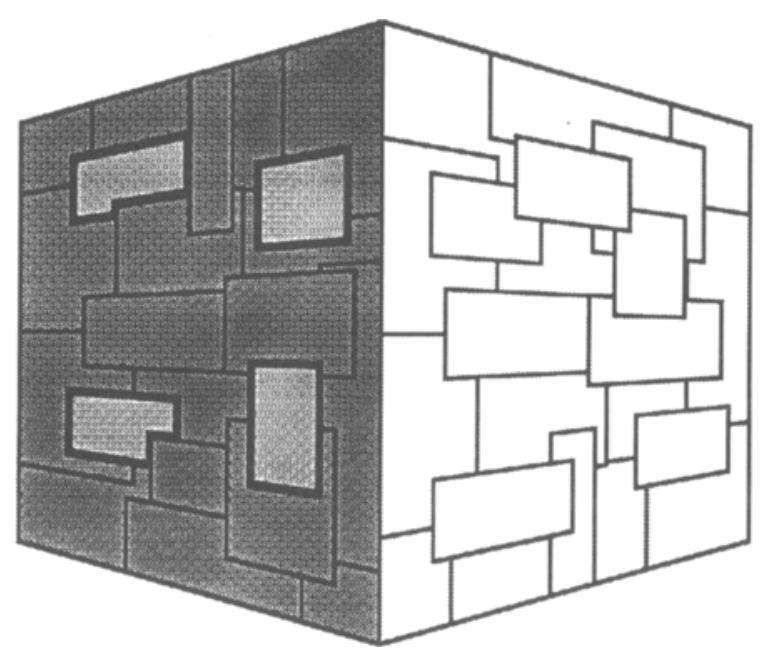

Figure 9. The visual field as seen by observers in Experiment 4. 
BACKGROUND LIGHTNESS

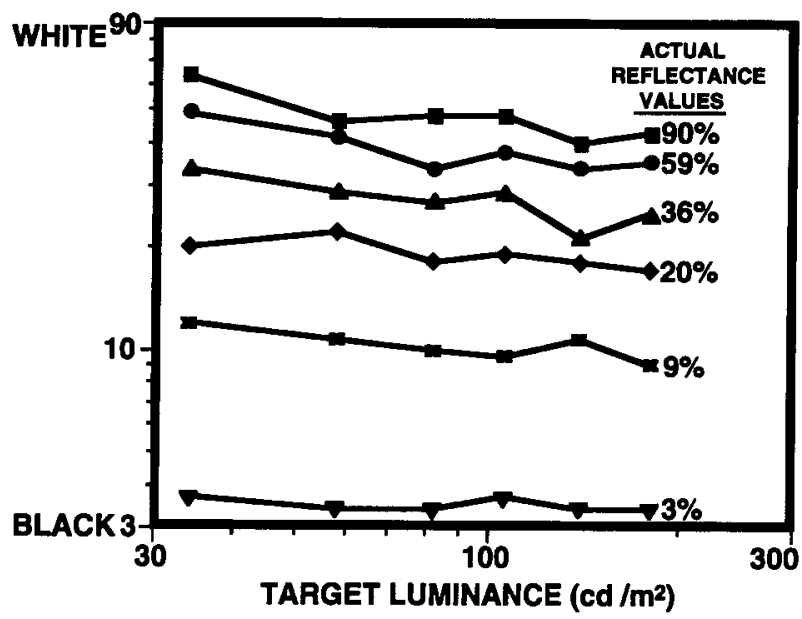

Figure 10. Lightness judgments for surfaces in the background of the targets in Experiment 4.

ducted under identical conditions using the same apparatus (Bonato \& Gilchrist, 1994). The luminosity threshold for the multiple targets presented in Experiment 4 was $118 \mathrm{~cd} / \mathrm{m}^{2}$, a value that is significantly higher $[t(28)=$ $3.0, p<.01]$ than $79 \mathrm{~cd} / \mathrm{m}^{2}$ (the threshold obtained for the single target in our earlier study).

The mean Munsell matches for the opaque surfaces on the Mondrian are shown in Figure 10. In general, as the luminance of the target regions increased, the perceived lightness values of the opaque surfaces on the Mondrian decreased. For all six surfaces tested, the mean lightness value for the highest target luminance condition was lower than that for the lowest target luminance condition. This difference reached significance, however, only for the three lightest shades $[F \mathrm{~s}(5,45)=6.2,6.0$, and 6.0 ; all $p s<.01]$.

The percentage of luminosity reports can be plotted as a function of the ratio between the luminance of the target and the luminance of a perceived white surface in the same illumination. The luminance value of a surface on the Mondrian that would be perceived as white was obtained by extrapolation, using the formula $W=(90 \% / R)$ $* L$, where $R$ is the reflectance of the mean Munsell match for a physical white surface and $L$ is the luminance of that surface. The data plotted in this manner, along with the data from our previous study using a single target, are shown in Figure 11. The two curves are quite similar, as are their threshold values.

The question remains as to whether the increased luminosity threshold was due to (1) the increased number of targets, from one to four, or (2) the increased aggregate surface area of the targets (about a fourfold increase). This was tested in Experiment 5.

\section{EXPERIMENT 5}

\section{Method}

The method used was identical to that used in Experiment 4 except that the four apertures were reduced in size such that their combined visual area was equal to that of a $2.3^{\circ}$ square, the same visual area of the single target aperture used by Bonato and Gilchrist (1994) in a previous experiment conducted under identical conditions.

\section{Results and Discussion}

In Experiment 5, we obtained a threshold value of $100 \mathrm{~cd} / \mathrm{m}^{2}$, which is not significantly different $[t(28)=$ 1.7] from the threshold value $\left(79 \mathrm{~cd} / \mathrm{m}^{2}\right)$ we obtained in our earlier experiment (Bonato \& Gilchrist, 1994) using a single target equal in area to the combined area of the four targets used here. So far, these results are consistent with the idea that the four targets exert their effect on the luminosity threshold by virtue of their aggregate area. However, the threshold obtained with the four small targets was not significantly different from that obtained with the four larger targets in Experiment 4. This provides a hint that there might be an effect of number of targets over and above the effect of aggregate area, but further work is called for.

\section{GENERAL DISCUSSION}

In these experiments, we have shown the following: (1) The luminosity threshold for a surface depends strongly on its area: The larger the surface, the higher the luminance at which the surface begins to appear selfluminous. (2) It is the perceived area that influences the luminosity threshold for a surface, not its retinal size.

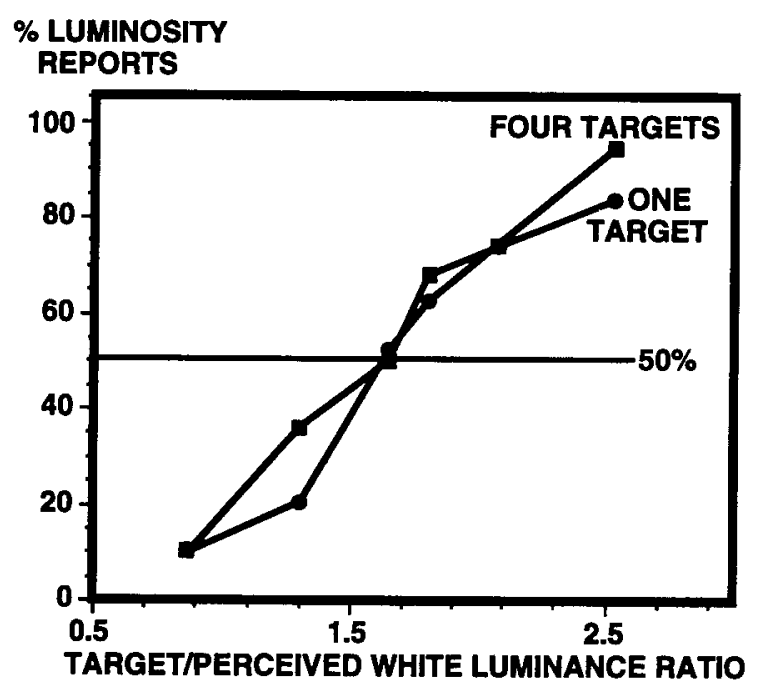

Figure 11. The percentage of luminosity reports for one target and four targets as a function of target:perceived-white luminance ratio (within the same plane). 
(3) Figure/ground does not affect the luminosity threshold directly, but it can affect the threshold indirectly by altering perceived area. (4) The luminosity threshold is higher for multiple targets of equal luminance than for a single target. However, it is likely that this effect is due to the aggregate area of the multiple targets rather than to their number.

We now discuss each of these points in more detail.

\section{The Luminosity Threshold Depends on Area}

This finding goes hand in hand with new evidence of the dependence of surface lightness on area. $\mathrm{Li}$ and Gilchrist (1999) have recently found that the anchoring of surface lightness depends not only on relative luminance but also on relative area, at least for simple displays. In addition to a tendency for the highest luminance in a framework to appear white, there is also a tendency for the largest area to appear white. Li and Gilchrist (1999) have formulated an area rule that describes perceived lightness values in simple, two-part visual frameworks: When the darker of two regions has the larger area, the lightness of the darker region varies directly with its perceived size. As the darker region grows in size, the lighter region is eventually forced into self-luminosity.

Note that this rule is not engaged when the lighter of two regions is also the larger, as in many displays (e.g., the familiar stimulus of a disk surrounded by a brighter annulus).

Note also that the conditions to which the area rule applies (darker region larger) are just the conditions under which anchoring by highest luminance and anchoring by largest area make conflicting demands on the percept. The rule describes how these conflicting demands are resolved. Anchoring by relative luminance is reflected in the fact that the higher of two luminances appears white. But anchoring by relative area is reflected in the fact that the darker (but larger) of the two regions moves a certain distance toward white. That is, it appears lighter in surface color than it would if its area were no greater than the area of the lighter region.

This conflict produces several strange results. First, it leads to a violation of the ratio principle in the sense that the ratio between the perceived lightness values (technically, the reflectance values of the two matching chips selected from a Munsell scale) of the two regions is less than the ratio between their physical reflectance values. The range of perceived lightness values is compressed relative to the range of luminance values. Second, either as the darker region becomes larger and larger or as its luminance becomes higher and higher, this violation of the ratio principle becomes more and more pronounced, causing the higher of the two luminances first to appear as a kind of superwhite and finally to appear as completely self-luminous. The superwhite appearance seems to be the same as both what Evans (1974, p. 99) referred to as fluorence and what Heinemann (1955) called the enhancement effect, in which increasing the luminance of an annulus causes a brightening of the surrounded disk, even though the disk has the higher luminance.
How severe a violation of the ratio principle can the visual system tolerate? Logically, there must be a limit, because there is a reductio ad absurdum point. According to the area rule, as the relative area of the darker region approaches that of the entire visual field, its lightness value must approach white. But if the lighter of the two regions merely continues to appear white (because it has the highest luminance), a point is reached at which the two regions must have the same lightness value (white) despite a large difference in their luminance values. It appears that this absurdity is avoided in the following way. Well before the reductio ad absurdum point is reached, the lighter region first begins to appear as a superwhite and finally as truly self-luminous.

This analysis is consistent with our findings on the luminosity threshold in the following way: The larger the relative size of the darker region (which means, of course, the smaller the relative size of the lighter region), the greater is the upward pressure on the lighter region to appear self-luminous. Thus, it is to be expected that a region perceived to have a smaller relative size will appear luminous more easily -that is, at a lower luminance level.

\section{The Luminosity Threshold Depends on Perceived Area, Not Retinal Area}

The distinction between a perceived and a retinal definition of stimulus factors such as area was developed into a powerful psychophysical tool by Irvin Rock (Rock \& Brosgole, 1964; Rock \& Ebenholtz, 1959). Our finding is consistent with Rock's general finding that key stimulus factors must be defined in perceptual, not retinal, terms. With regard to area, David Katz (1935) had embodied the distinction in his two laws of field size. According to his first law, the degree of lightness constancy depends on the retinal size of a region of special illumination (e.g., spotlight or shadow). According to the second law, constancy depends on the perceived size of the field of illumination. Katz thus regarded both definitions of size to be effective. To support the retinal definition, he observed that lightness constancy increases for surfaces seen through a smoked glass (neutral density filter) as it is drawn increasingly nearer to the eye (see Katz, 1935 , p. 218). But this demonstration is confounded in that perceived size varies along with retinal size. Thus, it may be that only Katz's second law is valid. Our results are consistent with such a conclusion.

The importance of perceived area over retinal area presents a serious challenge to the search for the physiological basis of the luminosity threshold, such as the possible role of receptive fields of different size.

\section{Figure/Ground Does Not Affect the Luminosity Threshold Directly, But It Can Affect the Threshold Indirectly by Altering Perceived Size}

Placing a figure on the target, as we did in Experiment 2, should make the target appear more ground-like. If figural regions appear self-luminous more readily than background regions, placing a figure on the target should raise its luminosity threshold. This did not occur. Con- 
sistent with this, Noguchi and Kozaki (1985) tested the perceived lightness of a dark gray disk in spotlight (Gelb effect) when two squares were placed on the disk so as to make the disk appear more ground-like. Two lighter squares caused the disk to appear darker gray, but two darker squares (black) had no effect. This suggests that, as long as the highest luminance is not changed, placing a figure on the target has no effect on its perceived lightness.

However, work done by Bonato and Cataliotti (1996) indicates that figure-ground relationship can affect the luminosity threshold when this in turn affects the perceived area of a region. They used a face-like profile to divide a rectangular region in half and tested the luminosity threshold for each of the two halves. Although the two halves had equal retinal area, they found a higher threshold value for the side that appeared as background, presumably because it appeared to extend behind the face side, giving it a larger perceived area.

More concretely, we can say that the functional area used to compute the luminosity threshold of a background region includes both its modal area and its amodal area. However, there is reason to believe that only a portion of the amodal area contributes to the functional area. Kanizsa (1979) has shown that there is a shrinkage of the amodal part of a surface that appears to extend behind an occluding region. Shimojo and Nakayama (1990), using an ambiguous apparent motion display, showed that the amodal extension of a partially occluded surface is not complete. This amodal shrinkage may explain why the target with the superimposed figure in Experiment 2 actually produced a slightly lower threshold than a homogeneous target of the same dimensions.

In a previous paper (Gilchrist \& Bonato, 1995), we noted that the very existence of perceived self-luminosity contradicts the highest luminance rule of anchoring, according to which the highest luminance in a display appears white (not luminous). We tried to resolve this contradiction by claiming that, in simple frameworks, the surround tends to appear white. However, our present results undermine such a surround-as-white rule. In Experiment 1 , the background appeared gray when a large high-luminance target was used. In Experiment 2, a background (in this case, the target) appeared luminous.

Because these findings were obtained using a relatively complex image, we could argue that the surround rule does not apply. However, the results we presented in our prior work (Gilchrist \& Bonato, 1995), interpreted there as supporting the surround-as-white rule are in fact consistent with the area rule described above. Also, given that our present findings are also consistent with this rule, we find it simpler to conclude that the surround rule is wrong. The area rule accounts very well for the data across a wide range of conditions.

\section{The Luminosity Threshold Depends on the Number of Targets}

The results of Experiments 4 and 5 do not make it clear whether the effect of number of targets is reducible to their combined area or whether number of targets is an effective factor in and of itself. Further work is called for here.

\section{Conclusions}

The results of these experiments reinforce the conclusion from our previous work (Bonato \& Gilchrist, 1994) that the luminosity threshold is tightly linked to the perception of surface lightness in general. In four separate conditions newly reported here, we obtained luminosity thresholds at target-to-perceived-white ratios between 1.6 and 1.8. These include the small and large target conditions of Experiment 1 and the four-target conditions of both Experiment 4 and Experiment 5 . We believe that this fixed distance between perceived white and the threshold of perceived luminosity suggests not only that the luminosity threshold is the border between opaque and luminous appearances but, further, that this border belongs to the lightness scale, not to the luminance scale, much as the border between a figure and its background belongs to the figure, not to the background.

The role of perceived area appears to provide the basic answer to the question of luminosity induction versus grayness induction. The larger the target, relative to its surround, the more the increased luminance difference is expressed as grayness induction of the surround (at the expense of luminosity induction of the target). The smaller the target, the more the increased luminance difference is expressed as luminosity induction (at the expense of grayness induction).

\section{REFERENCES}

Bonato, F., \& Cataliotti, J. (1996). Perceived area and figure/ground relations affect the luminosity threshold. Investigative Ophthalmology \& Visual Science, 37, s174.

Bonato, F., \& Gilchrist, A. L. (1994). The perception of luminosity on different backgrounds and in different illuminations. Perception, 23, 991-1006.

COREN, S. (1969). Brightness contrast as a function of figure-ground relations. Journal of Experimental Psychology, 80, 51 7-524.

DiAMOND, A. (1953). Foveal simultaneous brightness contrast as a function of inducing- and test-field luminances. Journal of Experimental Psychology, 45, 304-314.

Evans, R. M. (1974). The perception of color. New York: Wiley.

FREEMAN, R. B. (1967). Contrast interpretation of brightness constancy. Psychological Bulletin, 67, 165-187.

FRY, G. A., \& ALPERN, M. (1953). The effect of a peripheral glare source upon the apparent brightness of an object. Journal of the Optical Society of America, 43, 89-195.

GelB, A. (1929). Die "Farbenkonstanz" der Sehdinge [The "color constancy" of visual objects]. In W. A. von Bethe (Ed.), Handbuch der Normalen und Pathologischen Psychologie (Vol. 12, pp. 594-678). Berlin: Springer-Verlag.

Gilchrist, A. L., \& Bonato, F. (1995). Anchoring of lightness values in center/surround displays. Journal of Experimental Psychology: Human Perception \& Performance, 21, 1427-1440.

GOLDHAMmer, H. (1934). The influence of area, position, and brightness in the perception of a reversible configuration. American Journal of Psychology, 46, 186-206.

HeinemanN, E. G. (1955). Simultaneous brightness induction as a function of inducing- and test-field luminances. Journal of Experimental Psychology, 50, 89-96.

HoREMAN, H. W. (1965). Relations between brightness and luminance under induction. Vision Research, 5, 331-340. 
Kanizsa, G. (1979). Organization in vision. New York: Praeger.

KATZ, D. (1935). The world of colour. London: Kegan Paul, Trench, Trubner.

KÜNNAPAS, T. (1957). Experiments on figural dominance. Journal of Experimental Psychology, 53, 31-39.

Leibowitz, H., Mote, F. A., \& Thurlow, W. R. (1953). Simultaneous contrast as a function of separation between test and inducing fields. Journal of Experimental Psychology, 46, 453-456.

LI, X., \& Gilchrist, A. (1999). Relative area and relative luminance combine to anchor surface lightness values. Perception \& Psychophysics, 61, 771-785.

LIE, I. (1977). Perception of illumination. Scandinavian Journal of Psychology, 18, 251-255.

NoguchI, K., \& KozaKI, A. (1985). Perceptual scission of surfacelightness and illumination: An examination of the Gelb effect. Psychological Research, 47, 19-25.

OYama, T. (1950). Figure-ground dominance as a function of sector angle, brightness, hue, and orientation. Journal of Experimental Psychology, 60, 299-305.

ROCK, I., \& BROSGOLE, L. (1964). Grouping based on phenomenal proximity. Journal of Experimental Psychology, 67, 531-538.

Rock, I., \& EBENHOLTZ, S. (1959). The relational determination of perceived size. Psychological Review, 66, 387-401.

Shimojo, S., \& Nakayama, K. (1990). Amodal presence of partially occluded surfaces: Role of invisible stimuli in apparent motion correspondence. Perception, 19, 285-299.

SPEIGLE, J. M., \& BrainaRD, D. H. (1996). Luminosity thresholds: Effects of test chromaticity and ambient illumination. Journal of the Optical Society of America A, 13, 436-451.

TORII, S., \& UEMURA, Y. (1965). Effects of inducing luminance and area upon the apparent brightness of a test field. Japanese Psychological Research, 2, 86-100.
Ullman, S. (1976). On visual detection of light sources. Biological $C y$ bernetics, 21, 205-212.

WALLACH, H. (1948). Brightness constancy and the nature of achromatic colors. Journal of Experimental Psychology, 38, 310-324.

\section{NOTES}

1. We defined the luminosity threshold as the luminance at which the target appears luminous $50 \%$ of the time. Lie's (1977) instructions imply a higher degree of confidence, corresponding to a target that appears luminous perhaps $75 \%-100 \%$ of the time.

2. However, these observers did perceive the bright additional target as luminous. This indicates that their failure to report luminosity for any of the other targets was probably not due to a misunderstanding of instructions.

3. The fact that the illumination level in the matching apparatus was about 34 times higher than that in stimulus display constitutes a minor methodological weakness, because lightness can be contaminated, to some degree, by brightness, which, in this case, could slightly underestimate the matching Munsell values. Any error caused by this possible underestimation is likely to be quite small, and, in any case, it would affect the small target and large target conditions equally.

4. All of them, however, perceived the $676-\mathrm{cd} / \mathrm{m}^{2}$ target presented at the end of the experiment as luminous, which suggests that their failure to see any targets as luminous in the actual experiment was probably not due to a misunderstanding of instructions.

5. A schematic diagram of this vision tunnel is available in Bonato and Gilchrist (1994, p. 1001).

(Manuscript received March 17, 1997; revision accepted for publication March 19, 1998.) 